\title{
Motion tracking in young male football players: a preliminary study of within-session movement reliability
}

\author{
Mihkel M. Laas (id),a,d," Matthew D. Wright (id)a , Shaun J. McLaren (id) ${ }^{\mathbf{b}, \mathbf{c}}$, \\ Daniel L. Eaves $\mathbb{D}^{\mathbf{a}}$, Guy Parkin ${ }^{\mathbf{d}}$ and Matthew D. Portas ${ }^{\mathbf{a}, \mathbf{e}}$ \\ ${ }^{a}$ School of Health and Life Science, Teesside University, Middlesbrough, UK \\ ${ }^{b}$ Carnegie Applied Rugby Research Centre, Institute for Sport, Physical Activity and \\ Leisure, Leeds Beckett University, Leeds, UK \\ ${ }^{c}$ England Performance Unit, The Rugby Football League, Leeds, UK \\ ${ }^{d}$ Pro Sport Support Ltd, Huddersfield, UK \\ e Technical Directorate, The Football Association, St. George's Park, Burton upon Trent, \\ $U K$
}

${ }^{*}$ Corresponding author:

\section{Mr. Mihkel-Madis Laas, MSc}

School of Health and Life Science,

Teesside University,

Middlesbrough.

TS1 3BA.

UNITED KINGDOM.

Email:MM.Laas@tees.ac.uk

Telephone: +44 (0)7411314846 


\section{Disclosure of Funding}

The project received government funding from a Knowledge Transfer Partnership (Innovate UK) to Pro Sport Support Ltd and Teesside University (KTP 009965. Project title: To develop a specialist technology enhanced Adolescent Movement Analysis Tool and associated training intervention curriculum, exergaming and CPD offers underpinned by leading biomechanical research to improve the physicality of elite youth athletes). 


\begin{abstract}
We assessed the reliability of fundamental movement skills in young male footballers within one session. 197 players from 5 English category 3 football academies across U9 - U18 age groups volunteered (mean: age $=12.6 \pm 2.8$ years; stature $=156 \pm 17 \mathrm{~cm}$; weight $=47 \pm 15 \mathrm{~kg}$; years from peak height velocity $(\mathrm{PHV})$ $=-1.1 \pm 2.3$ ). Motion tracking of squat depth and anterior $\mathrm{Y}$-balance test maximum reach was recorded. Reliability was assessed (trial 1 vs. 2 ; trial 2 vs. 3) via mean change, intraclass correlation coefficient (ICC), and typical error. The overall mean change was trivial $(-1.8-2.9 \%)$, while Y-balance left showed a small negative change (trial 2 vs. $3 ;-3.2 \%$ ). All ICCs across the tests ranged from moderate to high (0.71 - 0.79). Typical errors for Y-balance tests ranged from $8.3-10.8 \%$ and squats from 19.3 - 21.8\%. Pre-PHV vs. post-PHV players had similar typical errors for Ybalance left and right (9.0 vs. $11.5 \%$; 9.0 vs. $7.1 \%$, respectively), and the squat (21.1 vs. $20 \%$ ). The high within-player typical error scores obtained over three trials suggest that, contrary to some movement screening guidelines, prior habituation is needed to increase both the stability and reliability of these tests.
\end{abstract}

Keywords: AMAT Performance; FMS; Movement screening; Movement variability; Reliability. 


\section{Introduction}

Fundamental movements, such as squatting and balancing are important for the physical development of children and adolescents, as they provide the building blocks for more advanced training (Hulteen, Morgan, Barnett, Stodden, \& Lubans, 2018; Lubans, Morgan, Cliff, Barnett, \& Okely, 2010), and have biomechanical similarities to sport-specific movements (Tompsett, Burkett, \& McKean, 2014). Screening batteries that involve performing and assessing fundamental movements are widespread in the fields of sports medicine and athletic performance (Bishop, Read, Walker, \& Turner, 2015; Gamble, 2013). The most common tool for testing fundamental movement patterns in high level professional association football is the Functional Movement Screen (FMS ${ }^{\text {TM}}$; McCall et al. 2014), which is also widely used with youth athletes (Newton et al. 2017; Portas, Parkin, Roberts, \& Batterham, 2016; Lloyd et al. 2015). The purpose of the FMS ${ }^{\mathrm{TM}}$ is to screen athletes for dysfunctional movement patterns in an attempt to reduce injury risk and ensure safety in training (Cook, Burton, Kiesel, Rose, \& Bryant, 2010; Cook, Burton, \& Hogenboom, 2006a; Cook, Burton, \& Hogenboom, 2006b). Crucially, the FMS ${ }^{\text {TM }}$ guidelines recommend three trials to assess each of the seven fundamental movement skills. The guidelines also advise that a warm-up should not be undertaken, to allow assessment of the participant's natural state of movement. This method is considered the best indication of the quality and level of movement competency experienced in the person`s current daily activity (Cook, 2015).

Like most movement screening tools (e.g., McCunn et al. 2017; McKeown, Taylor-McKeown, Woods, \& Ball, 2014), the FMS ${ }^{\mathrm{TM}}$ uses a categorical scaling system to quantify movement quality. The criteria are based on the identification of biomechanical and neuromuscular deficits that hinder optimal performance and pose 
a risk of injuries (Bishop et al. 2015; Kushner et al. 2015; McKeown et al. 2014; Cook et al. 2006a). A criticism of these tools is that the inter-rater reliability is dependent on trained testers (Shultz, Anderson, Matheson, Marcello, \& Besier, 2011; Minick et al. 2010) and subjective judgements made when determining the outcome score. Crude categorical scaling reduces the likelihood of successfully identifying a practically important change in performance. For example, a minimum practically important difference for these scales probably lies between 0.5 and 1 arbitrary unit (Wright \& Chesterton, 2018; Hopkins, 2010) which makes establishing improvement extremely difficult on a 0-3 point scale.

Traditional methods of assessing fundamental movements in athletes do not consider the potential influence of biological variability on the outcome score. For example, in the FMS" movement quality is determined based on a single or, "best repetition", whereas consistency of performance is not taken into account. While variability was traditionally seen as "noise" that should be eliminated via practice (Preatoni et al. 2013), the inherent variation across multiple repetitions of a single task helps performers adapt to new environments, reduce injury risk and can facilitate motor learning (Bartlett, Wheat, \& Robins, 2007; Stergiou, Harbourne, \& Cavanaugh, 2006). Variability during the execution of athletic movements can increase with expertise, while keeping the outcome variability low (Hamacher \& Zech, 2018; Yeadon \& Hiley, 2014). Variability is therefore a core functional component of human movement which may be overlooked and unaccounted for when using traditional methods for assessing fundamental movements.

In an attempt to reduce inter-tester reliability issues and increase the objectivity of fundamental movement skill testing, a novel assessment tool and testing battery called AMAT Performance (Pro Sport Support Ltd), was used in the 
present study. The AMAT Performance involves movement recordings via a portable and bespoke motion tracking system using the Microsoft Xbox Kinect camera. Recent assessments have found this system provides good criterion validity for athletic movements against manual concurrent measurements (McLaren et al. under review). The system`s marker-based tracking and calibration algorithms also produced acceptable reliability with multiple camera and trial measurements. The average differences in frame-to-frame $(1.3 \mathrm{~mm})$, trial-to-trial $(0.1 \mathrm{~mm})$ and camerato-camera $(4 \mathrm{~mm})$ measurements were sufficiently low (McLaren et al. under review). Since the scoring system is interval-ratio level, this system offers greater precision in assessments, compared to traditional movement screening batteries, providing objective scores and the ability to detect between-trial and betweensession variability both within a single athlete, and between groups of athletes.

As a preliminary step, the aim of the present study was to assess the reliability in outcome scores for fundamental movements during a single session in young male football players. The objectives were to determine the reliability of the tests, to describe the trial-to-trial, within-subject variability (Voisin, Jacques, Lucia, Bishop, \& Eynon, 2019) and to explore if the common practice of using 3 trials per test, in the absence of a warm-up is sufficient for the stabilisation of the scores for these athletic movement tests. 


\section{Methods}

Design

This study was designed to measure within-session reliability of two fundamental athletic movements (balance and squat) in trained young male football players. The experiment therefore incorporated a test-retest research design.

\section{Participants}

A total of 197 young male footballers from five English category three football club academies within the English Elite Player Performance Plan (EPPP) system took part in this study. The final sample included players from the U9 to U18 age groups (mean age: $12.6 \pm 2.8$ years; height: $156 \pm 17 \mathrm{~cm}$; sitting height: $80 \pm 9$ cm, weight: $47 \pm 15 \mathrm{~kg}$ ). Biological age was calculated via maturity offset: $-1.1 \pm$ 2.3 years from Peak Height Velocity (PHV) (Mirwald, Baxter-Jones, Bailey, \& Beunen, 2002). The player distribution according to chronological age and maturation groups for the anterior Y-balance and back squat tests can be seen on Figure 1. All players were injury-free and medically cleared to participate in training by the clubs medical staff. Ethical release was obtained from Teesside University's Research Ethics committee to use anonymised data provided by Pro Sport Support Ltd.

***FIGURE 1 ABOUT HERE*** 


\section{Experiment}

To investigate the within session reliability of the movement tests, the players completed three trials of either the anterior Y-balance or back squat test on the AMAT Performance system during their 2017/18 pre-season biomechanical testing battery. A schematic of the sample and participant characteristics for each test can be seen in Figure 1. The movement scores were tracked with a $30 \mathrm{~Hz}$ depth sensor camera (Kinect ${ }^{\mathrm{TM}}$ V2, Microsoft, USA). Some of the trials were excluded when the complete movements were not successfully tracked by the system (e.g., due to performing the tests too early or late according to the recording time; Ybalance: $n=23$; back squat: $n=27$ ). The testing took place in an indoor environment at each club (e.g., sports hall, gym), where the AMAT Performance system was set up. The players were asked to wear their normal indoor training footwear and a reflective marker was attached to the middle of their shoe laces and above the patella, as per the manufacturer's user guidelines. Players were given verbal instructions on how to perform the movements of the balance or the squat beforehand, and received a demonstration of the tests on the AMAT Performance system by trained staff.

In line with previous guidelines for the assessment of fundamental movements (Cook, 2015), no warm-up or habituation period was provided prior to testing. The tests started and finished with an auditory cue from the AMAT Performance system, which was explained to the players beforehand. The balance slider (Figure 2(a)) and the squat saddle (Figure 2(c)) of the system were used for measurement distance of the tests (Figure 2(b,d)). 
For the anterior Y-balance tests on the first audio cue the players were instructed to sit back on the standing leg and using their free limb in a controlled manner, push the slider as far as possible whilst counting to three (Figure 2(b)). They were then asked to pull the leg back and balance at the end until the second audio cue. No restriction was placed on the amount of hip, knee or ankle flexion of the standing foot. Balance left referred to the standing leg and vice versa for balance right, as recommended in the Y-balance testing protocol (Plisky et al. 2009). For the back squat test the players were asked to stand with feet shoulder width apart holding a dowel against their back. On the first audio cue they were instructed to sit back against the squat saddle and squat as deep as possible in a controlled manner whilst counting to three, then to stand up and remain still until the second audio cue (Figure 2(d)). The players were informed to keep their feet flat on the floor throughout the flexion and extension of the hip, knee and ankle. Similar instructions have been proposed before for back squat assessment (Myer et al. 2014). These instructions are used with the AMAT Performance system to encourage the athletes to complete the movements during the recording time. The motion tracking lasted 5 seconds per trial for both the balance and squat tests, during which 150 frames of data were recorded. A maximum movement performance outcome score was recorded indicating the distance where the players pushed the balance slider in the balance tests (Plisky et al. 2009) and the squat saddle in the squat test. The anterior Y-balance performance was measured as the foremost position of the balance slider marker from the start position (mm, measured in the z-axis). The squat performance was measured tracking the squat saddle marker, as the marker`s maximum displacement of the 
movement from the start to the bottom position ( $\mathrm{mm}$, measured in the y-axis). For the Y-balance test, the players performed all their trials on the left side and then on the right side according to the AMAT Performance testing protocol.

\section{Statistical analysis}

Raw data for the balance and back squat scores were summarised as the mean ( \pm standard deviation $[\mathrm{SD}]$ ) for the overall dataset and also the median (minimum and maximum) for each separate age group. Prior to analysis, all data were logtransformed to reduce bias associated with non-uniformity of error and were subsequently back-transformed to obtain reliability statistics in percentage units (as well as raw units). Paired samples $t$-tests were used to evaluate the systematic bias as the mean difference between trials (trial 2 vs. trial 1, trial 3 vs. trial 2). Random error was assessed using the typical error (i.e. within-player variation), estimated as the SD of change scores divided by the square root of 2 (Hopkins, 2000). We also assessed random error using the intraclass correlation coefficient (ICC; i.e. reproducibility of the rank order of players). The ICC was calculated from a 2-way mixed-effects model $\left(\mathrm{ICC}_{3,1}\right.$; Shrout \& Fleiss, 1979). Uncertainty in all estimates were shown as $90 \%$ confidence intervals throughout. Confidence limits for the typical error were calculated using the chi-squared distribution and confidence limits for the ICC were calculated using an F-distribution.

The magnitude of the between-trial changes were interpreted using standardised thresholds for trivial, small, moderate and large differences of $<0.2,0.2$, 0.6 and 1.2 of the pooled between-subject SD (Hopkins, Marshall, Batterham, \& Hanin, 2009). To assess the magnitude of typical error, these thresholds were halved 
(Atkinson \& Batterham, 2015). The observed between-trial reductions in typical errors were considered meaningful when they crossed a magnitude threshold (e.g. "moderate" to "small"; Hurst, Batterham, Weston, \& Weston, 2018). The qualitative inference for the $\mathrm{ICC}_{3,1}$ was based on the following thresholds: $>0.99$, extremely high; $0.99-0.90$, very high; $0.75-0.90$, high; $0.50-0.75$, moderate; $0.20-0.50$, low; $<0.20$, very low (Malcata, Vandenbogaerde, \& Hopkins, 2014).

\title{
Results
}

Descriptive statistics for the movement tests

Individual and age group descriptive movement performance data are presented in Figure 3 and Table 1.

\author{
***FIGURE 3 ABOUT HERE*** \\ ***TABLE 1 ABOUT HERE***
}

\section{Within-session reliability}

The mean changes were under 3\% and rated trivial, except for anterior $\mathrm{Y}$ balance left (trial 3 vs. 2) which had a small decrease. The overall typical errors were around $10 \%$ for anterior Y-balance left, $8 \%$ for the right balance side and $20 \%$ for the back squat. The typical errors were higher between trials 3 to 2 compared to trials 2 to 1 for anterior Y-balance test both legs and lower for the back squat. The ICCs 
ranged from 0.75 to 0.79 for all the tests, except between anterior Y-balance left (trial 3 vs. 2) which was 0.71 (Table 2).

\section{***TABLE 2 ABOUT HERE***}

\section{Variability across maturation}

The typical errors across maturation groups were similar, with the post-PHV group having slightly lower overall typical errors against pre-PHV group on the anterior Y-balance right (7.1\% vs. 9.0\%), and back squat (20.0\% vs. $21.1 \%)$, but higher on the anterior Y-balance left $(11.5 \%$ vs. 9.0\%) (Figure 4). The only reduction of typical errors across meaningful thresholds between maturation groups came from the pre-PHV group in the back squat, which changed from large to moderate (Figure 4).

***FIGURE 4 ABOUT HERE***

\section{Discussion}

Movement screening is common place in athletic populations, yet the degree to which test performance stabilises with repeated assessments is an important but often overlooked issue. The aim of this study was to assess the within-session reliability of fundamental athletic movements in trained young male football players. Our data are the first to quantify the trial-to-trial reliability of test performance in the 
anterior Y-balance and back squat tests from a large male population. To the best of our knowledge we also made the first quantification of movement performance outcome variability of the selected tests in young boys` football cohorts. We report trivial mean changes, moderate to high ICCs, and moderate to large typical errors (within-subject variation) which were similar regardless of maturation status. The primary finding was that three trials were insufficient to provide a stable measure of performance in either of the tests, contrary to current movement screening guidelines (Cook, 2015), which advocate only three trials without either a warm-up or habituation period.

We did not provide evidence for a systematic change in the anterior $\mathrm{Y}$ balance and back squat tests across 3 trials. Systematic change, represented as a change in the mean, refers to a meaningful performance change at the group level (Hopkins, 2000). The trial-to-trial mean changes were trivial, except for Y-balance left, which showed a small reduction. The typical errors (Table 2) were moderate to large indicating that the random error was high. The typical errors increased across the trials (trial 2 vs. trial 1 and trial 3 vs. trial 2) for both the left and right Y-balance tests $(8.8-10.8 \% ; 8.3-8.7 \%)$. The typical errors were larger during the back squat test, but decreased slightly with trial comparison $(21.8 \%-19.3 \%)$. The ICCs, which represent the extent an individual maintains their position in a sample with repeated trials (Batterham \& George, 2000), ranged from moderate to high (0.71-0.79) across all the trials and tests (Table 2). Traditionally six practice trials and three test trials are recommended for Y-balance testing (Plisky et al. 2009), but Robinson and Gribble (2008) showed that the anterior Y-balance scores reached stability with four practice trials. Both studies (Plisky et al. 2009; Robinson \& Gribble, 2008) used adult participants in their protocols. Robinson and Gribble (2008) also had the 
participants undertaking the test barefooted, which might have influenced the scores. Due to the unique squatting mechanism used in our testing protocol (Figure 2(c)) previous research does not inform on the number of trials needed to reach stability of the back squat. The results of our study do indicate, however, that due to high intraindividual variability further exploration would be required to empirically establish the number of trials required to achieve stabilisation of the outcome scores for the tests.

Our findings are similar to previous research regarding the anterior balance test-re-test reliability in children, which were also moderate to high (Calatayud, Borreani, Colado, Martin, \& Flandez, 2014; Faigenbaum et al. 2014). As in our study, Calatayud and colleagues (2014) observed left stance balance to be less reliable (moderate) and had a larger typical error. Faigenbaum and colleagues (2014) also demonstrated high ICCs for both legs and typical error in left and right legs of 3.5-3.4 cm compared to our 5.5-4.9 $\mathrm{cm}$ (Table 2). Although our players achieved higher scores on the left compared to right Y-balance test (Table 1), they also displayed greater within-subject variability on the left leg (Table 2). Higher movement outcome variability has been linked with inferior skill levels (Hamacher \& Zech, 2018; Yeadon \& Hiley, 2014; Hiley, Zuevsky, Maurice, \& Yeadon, 2013; Nakayama, Kudo, \& Ohtsuki, 2010), which would be contrary to previous research regarding the non-dominant leg, with the left leg typically being more stable in football players (Barone et al. 2011). This information could be useful for strength and conditioning coaches, who should take into account within-player differences for each leg when assessing and developing balance in football.

Our study showed little differences in the intra-individual variability in the tests between maturation groups. The left and right Y-balance typical errors 
increased from trial 2 to 1 and 3 to 2 for both maturation groups. Unlike on the Ybalance right, the overall typical errors for the Y-balance left were lower on the prePHV compared to post-PHV group. The overall typical errors were higher in the prePHV compared to post-PHV in the back squat, but decreased with successive trials (Figure 4). An interesting finding was that the overall typical errors for Y-balance left and right remained stable for pre-PHV $(\sim 9 \%)$, whereas the post-PHV group showed more variability on the left leg $(\sim 12 \%)$ compared to the right $(\sim 7 \%)$ (Figure 4). The only observation of the typical errors crossing the magnitude threshold came from the pre-PHV group in the back squat test, reducing the typical error by trial three from large to moderate (Figure 4). Children at pre-PHV might therefore have an advantage in terms of mastering fundamental movement skills at a faster rate due to increased neuroplasticity (Myer et al. 2015) as reflected in the Youth Development Model, which states that the ideal time to develop fundamental movement skills is during prepubescent period (Lloyd \& Oliver, 2012).

Movement outcome variability existing between the trials of the tests is likely biological in this study and not technical, as trial-to-trial system variability is $\sim 0.1 \mathrm{~mm}$ for the AMAT Performance system (McLaren et al. under review). Variability in movement outcomes have been investigated previously (Hamacher \& Zech, 2018; Yeadon \& Hiley, 2014; Hiley et al. 2013; Nakayama et al. 2010) and shown to be low in experts, but less research is available on children. We would have expected more mature players to be more consistent due to increased training age and based on previous findings that more mature players achieve higher outcome performance on fundamental movement assessments (Ryan et al. 2018; Portas et al. 2016). Both the balance and squat tests are dependent on stability, where more mature players have previously outperformed their less mature counterparts (Wright 
\& Chesterton, 2018; Portas et al. 2016). The values we report here for typical error, however, were moderate-to-large for both maturation groups. This suggests that an additional focus on developing fundamental movement skills would be beneficial throughout the different stages of maturation. Practitioners should therefore explore novel training strategies for developing fundamental movement outcome performance and its consistency.

A few limitations need to be considered when interpreting our findings. First, the method of assessing maturation via maturity offset (Mirwald et al. 2002) has previously been questioned due to its associated error of \pm 6 months in boys. However, the equations used have previously been found to be reliable (Mills, Baker, Pacey, Wollin, \& Drew, 2016). Secondly, because of the low numbers in the age groups (Table 1), the results may not be a perfect representation of each chronological age population, and this could potentially increase the likelihood of type one and two errors with the smaller groups (Knudson \& Lindsey, 2014). We were reliant, however, on anonymised data and could not carefully scrutinize the group sizes for this study. The latter rationale also accounts for why we could not control for potential bias towards pre-PHV children and players undertaking the anterior Y-balance test (Figure 1). Thirdly, our purpose was to test young male football players as we only had access to their data in this study. Considering that there are slight differences in the squat and anterior reach scores between gender (Butler, Elkins, Kiesel, \& Plisky, 2009; Plisky, Rauh, Kaminski, \& Underwood, 2006) the conclusions in this study would be applicable to boy`s cohort at this time. Lastly, the applied training environment might not be ideal for movement screening as there are constraints inherent in testing large groups with limited time. To mitigate this issue, the AMAT system we employed was developed to bring sophisticated 
technology into the football academies. While physical testing in this environment has previously been found to be reliable alongside with the daily football academy routine (Enright et al. 2018), we cannot rule out that this might have influenced the high intra-individual variability in the players`scores.

\section{Conclusions}

Our study showed that there was no substantial change in the mean fundamental movement scores from trial-to-trial, but there was a moderate-to-large variability within the players themselves across the trials. The reliability of the ICCs ranged from moderate to high across the tests and trials. We conclude that the squat and balance performance did not stabilise over three trials in young male football players. The common movement screening guidelines (Cook, 2015) for assessing fundamental movements in the natural state of movement therefore need to be interpreted with caution. Assessing the stability and consistency of movement outcome variables is important when monitoring young male football players.

\section{Acknowledgements}

We would like to express our gratitude to Professor Alan M. Batterham for his invaluable statistical advice throughout the completion of this research. The authors acknowledge the help of Pro Sport Support Ltd for the provision of the anonymised data, which was used for this study. At the time of this research, MML and GP were employees of Pro Sport Support Ltd-a company seeking the development and commercial sale of practical, marker based tracking systems for 
athletic movement screening. The project received government funding from a Knowledge Transfer Partnership (Innovate UK) to Pro Sport Support Ltd and Teesside University (KTP 009965).

\section{Disclosure statement}

No potential conflict of interest was reported by the authors.

\section{ORCID}

Mihkel M. Laas (D) https://orcid.org/0000-0002-2401-1092

Matthew D. Wright (iD) http://orcid.org/0000-0002-4909-4162

Shaun J. McLaren (id http://orcid.org/0000-0003-0480-3209

Daniel L. Eaves (D) https://orcid.org/0000-0003-2436-7694 


\section{References}

Atkinson G, Batterham AM. 2015. True and false interindividual differences in the physiological response to an intervention. Exper Phys. 100(6):577-588.

Barone R, Macaluso F, Traina M, Leonardi V, Farina F, Di Felice V. 2011. Soccer players have a better standing balance in nondominant one-legged stance. $\mathrm{J}$ Sports Med. 2:1-6.

Bartlett R, Wheat J, Robins M. 2007. Is movement variability important for sports biomechanists? Sports Biomec. 6(2):224-243.

Batterham AM, George KP. 2000. Reliability in evidence-based clinical practice: A primer for allied health professionals. Phys Ther Sport. 1(2):54-62.

Bishop C, Read P, Walker S, Turner A. 2015. Assessing movement using a variety of screening tests. Prof Strength Cond. 37(6):17-26.

Butler RJ, Elkins B, Kiesel KB, Plisky PJ. 2009. Gender Differences in Functional Movement Screen and Y-balance Test Scores in Middle School Aged Children. Med Sci Sports Exerc. 41(5):183.

Calatayud J, Borreani S, Colado JC, Martin F, Flandez J. 2014. Test-Retest Reliability of the Star Excursion Balance Test in Primary School Children. The Phys Sportsmed. 42(4):120-124.

Cook G, Burton L, Hogenboom B. 2006a. Pre-Participation Screening: The Use of Fundamental Movements as an Assessment of Function - Part 1. N Am J Sports Phys Ther . 1:62-72.

Cook G, Burton L, Hogenboom B. 2006b. Pre-Participation Screening: The Use of Fundamental Movements as an Assessment of Function - Part 2. N Am J Sports Phys Ther. 1:132-139.

Cook G, Burton L, Kiesel K, Rose G, Bryant MF. 2010. Movement: Functional Movement Systems: Screening, Assessment, Corrective Strategies.

Cook G. 2015. The FMS ${ }^{\text {TM }}$ Functional Movement Screen Level 1 Online Version 2. Functional Movement Systems, p.6.

Enright K, Morton J, Iga J, Lothian D, Roberts S, Drust B. 2018. Reliability of "in-season" fitness assessments in youth elite soccer players: a working model for practitioners and coaches. Sci Med Football. 2(3):177-183.

Faigenbaum AD, Myer GD, Fernandez IP, Carrasco EG, Bates N, Farrell A, Ratamess NA, Kang J. 2014. Feasibility and reliability of dynamic postural control measures in children in first through fifth grades. Int J Sports Phys Ther. 9(2):140148. 
Gamble P. 2013. Movement screening protocols: Rationale versus evidence. NZ J Sports Med. 40:83-87.

Hamacher D, Zech A. 2018. Development of functional variability during the motor learning process of a complex cyclic movement. J Biomech. 77:124-130.

Hiley MJ, Zuevsky VV, Maurice R, Yeadon MR. 2013. Is skilled technique characterized by high or low variability? An analysis of high bar giant circles. Hum Mov Sci. 32:171-180.

Hopkins WG, Marshall SW, Batterham AM, Hanin J. 2009. Progressive statistics for studies in sports medicine and exercise science. Med Sci Sports Exerc. 41(1):3-13.

Hopkins WG. 2000. Measures of reliability in sports medicine and science. Sports Med. 30(1):1-15.

Hopkins WG. 2010. Linear Models and Effect Magnitudes for Research, Clinical and Practical Applications. Sport Sci. 14:49-58.

Hulteen RM, Morgan PJ, Barnett LM, Stodden DF, Lubans DR. 2018. Development of Foundational Movement Skills: A Conceptual Model for Physical Activity Across the Lifespan. Sports Med. 48:1533-1540.

Hurst C, Batterham AM, Weston KL, Weston M. 2018. Short- and long-term reliability of leg extensor power measurement in middle-aged and older adults. $\mathrm{J}$ Sports Sci. 36(9):970-977.

Knudson DV, Lindsey C. 2014. Type I and Type II erros in correlations of various sample sizes. Compr Psych. 3(1):1-5.

Kushner AM, Jensen LB, Schoenfeld BJ, Hugentobler J, Lloyd RS, Vermeil A, Chu DA, Harbin J, McGill SM, Myer GD. 2015. The Back Squat: Targeted Training Techniques to Correct Functional Deficits and Technical Factors That Limit Performance. Strength Cond J. 37(2):13-60.

Lloyd RS, Oliver JL, Radnor JM, Rhodes BC, Faigenbaum AD, Myer GD. 2015. Relationships between functional movement scores, maturation and physical performance in young soccer players. J Sports Sci. 33(1):11-19.

Lloyd RS, Oliver JL. 2012. The Youth Physical Development Model: A New Approach to Long-Term Athletic Development. Strength Cond J. 34(3):61-72.

Lubans DR, Morgan PJ, Cliff DP, Barnett LM, Okely AD. 2010. Fundamental movement skills in children and adolescents: review of associated health benefits. Sports Med. 40(12):1019-1035. 
Malcata RM, Vandenbogaerde TJ, Hopkins WG. 2014. Using athletes' World rankings to assess countries' Performance. Int J Sports Physiol Perform. 9(1):133138.

McCall A, Carling C, Nedelec M, Davison M, Le Gall F, Berthoin S, Dupont G. 2014. Risk factors, testing and preventative strategies for non-contact injuries in professional football: current perceptions and practices of 44 teams from various premier leagues. Br J Sports Med. 48(18):1352-1357.

McCunn R, Fünten K, Govus A, Ross J, Schimpchen J, Meyer T. 2017. The intra- and inter-rater reliability of the soccer injury movement screen (SIMS). Int $J$ Sports Phys Ther. 12(1):1-14.

McKeown I, Taylor-McKeown, Woods C, Ball N. 2014. Athletic Ability Assessment: A movement assessment protocol for athletes. Int J Sports Phys Ther. 9(7):862-873.

McLaren SJ, Wijnbergen M, Portas MD, Weston M, Telford J, Laas MM, Parkin G, Spears IR. Validity and reliability of a video-based system for the automated measurement of performance during athlete movement tests. Manuscript under review.

Mills K, Baker D, Pacey V, Wollin, M, Drew MK. 2016. What is the most accurate and reliable methodological approach for predicting peak height velocity in adolescents? A systematic review. J Sci Med Sport. 20(6):1-6.

Minick KI, Kiesel KB, Burton L, Taylor A, Plisky P, Butler RJ. 2010. Interrater reliability of the functional movement screen. J Strength Cond Res. 24(2):479-486.

Mirwald RL, Baxter-Jones ADG, Bailey DA, Beunen GP. 2002. An assessment of maturity from anthropometric measurements. Med Sci Sports Exerc. 34(4):689-694.

Myer GD, Faigenabaum AD, Edwards NM, Clark JF, Best TM, Sallis RE. 2015. Sixty minutes of what? A developing brain perspective for activating children with an integrative exercise approach. Br J Sports Med. 0:1-9.

Myer GD, Kushner AM, Jensen BL, Schoenfeld BJ, Hugentobler J, Lloyd RS, Vermeil A, Chu DA, Harbin J, McGill SM. 2014. The Back Squat: A Proposed Assessment of Functional Deficits and Technical Factors That Limit Performance. Strength Cond J. 36(6):4-27.

Nakayama Y, Kudo K, Ohtsuki T. 2010.Variability and fluctuation in running gait cycle of trained runners and non-runners. Gait Posture. 3:331-335.

Newton F, McCall A, Ryan D, Blackburne C, aus der Fünten K, Meyer T, Lewin C, McCunn R. 2017. Functional Movement Screen (FMS ${ }^{\mathrm{TM}}$ ) score does not 
predict injury in English Premier League youth academy football players. Sci Med Football. 1(2):102-106.

Plisky PJ, Rauh MJ, Kaminski TW, Underwood FB. 2006. Star Excursion Balance Test as a predictor of lower extremity injury in high school basketball players. J Orthop Sports Phys Ther. 36(12):911-919.

Plisky PJ, Gorman PP, Butler RJ, Kiesel KB, Underwood FB, Elkins B. 2009. The Reliability of an Instrumented Device for Measuring Components of the Star Excursion Balance Test. N Am J Sports Phys Ther. 4(2):92-99.

Portas MD, Parkin G, Roberts J, Batterham AM. 2016. Maturational effect on Functional Movement Screen ${ }^{\mathrm{TM}}$ score in adolescent soccer players. J Sci Med Sport. 19(10):854-858.

Preatoni E, Hamill J, Harrison AJ, Hayes K, Van Emmerik RE, Wilson C, Rodano R. 2013. Movement variability and skills monitoring in sports. Sports Biomech. 12(2):69-92.

Robinson RH, Gribble G. 2008. Support for a Reduction in the Number of Trials Needed for the Star Excursion Balance Test. Arch Phys Med Rehabil. 89(2):364-370.

Ryan D, McCall A, Fitzpatrick G, Hennessy L, Meyer T, McCunn R. 2018. The influence of maturity status on movement quality among English Premier League academy soccer players. SPSR. 32:1-3.

Shrout PE, Fleiss JL. 1979. Intraclass Correlations: Uses in Assessing Rater Reliability. Psychol Bull. 86(2):420-428.

Shultz R, Anderson S, Matheson GO, Marcello B, Besier T. 2011. Functional movement screen: Inter-rater and subject reliability. Br J Sports Med. 45:374.

Stergiou N, Harbourne RT, Cavanaugh JT. 2006. Optimal movement variability: A new theoretical perspective for neurologic physical therapy. J Neurol Phys Therap. 30:120-129.

Tompsett C, Burkett B, McKean MR. 2014. Development of Physical literacy and movement competency: A literature review. J Fit Res. 3(2):53-74.

Voisin S, Jacques M, Lucia A, Bishop DJ, Eynon N. 2019. Statistical considerations for exercise protocols aimed at measuring trainability. Exerc Sport Sci Rev. 47(1):37-45.

Wright MD, Chesterton P. 2018. Functional Movement Screen ${ }^{\mathrm{TM}}$ total score does not present a gestalt measure of movement quality in youth athletes. J Sports Sci. 24:1-10. 
Yeadon MR, Hiley MJ. 2014. The control of twisting somersaults. J Biomech. 47(6):1340-1347. 


\section{TABLES}

Table 1. Descriptive statistics comparison between age groups for the anterior Ybalance and back squat tests.

\begin{tabular}{|c|c|c|c|c|c|}
\hline Test and age group & Mean & \pm SD & Median & Maximum & Minimum \\
\hline \multicolumn{6}{|c|}{$\begin{array}{l}\text { Anterior Y-balance Left } \\
\text { (cm) }\end{array}$} \\
\hline U9 & 56.3 & 9.0 & 55.4 & 82.6 & 37.7 \\
\hline U10 & 60.6 & 10.1 & 62.4 & 80.3 & 32.6 \\
\hline U11 & 58.2 & 8.6 & 57.1 & 76.6 & 40.5 \\
\hline U16 & 74.2 & 4.9 & 74.0 & 83.8 & 65.2 \\
\hline U18 & 62.1 & 11.7 & 62.8 & 84.4 & 34.9 \\
\hline Overall & 60.6 & 10.5 & 60.3 & 84.4 & 32.6 \\
\hline \multicolumn{6}{|l|}{$\begin{array}{l}\text { Anterior Y-balance } \\
\text { Right (cm) }\end{array}$} \\
\hline U9 & 53.2 & 9.3 & 53.8 & 69.5 & 27.5 \\
\hline U10 & 59.4 & 9.0 & 60.0 & 84.7 & 35.4 \\
\hline U11 & 57.0 & 8.0 & 57.4 & 72.4 & 41.0 \\
\hline U16 & 75.8 & 4.6 & 75.6 & 82.0 & 65.3 \\
\hline U18 & 59.7 & 10.7 & 61.3 & 75.7 & 37.7 \\
\hline Overall & 58.6 & 10.2 & 58.6 & 84.7 & 27.5 \\
\hline \multicolumn{6}{|l|}{ Back squat (cm) } \\
\hline U9 & 42.3 & 6.7 & 45.5 & 46.9 & 21.5 \\
\hline U10 & 38.0 & 8.3 & 39.1 & 46.4 & 9.4 \\
\hline U11 & 38.5 & 7.8 & 39.1 & 47.1 & 18.9 \\
\hline U12 & 34.7 & 7.8 & 35.6 & 47.0 & 12.4 \\
\hline U13 & 37.3 & 13.3 & 40.3 & 51.9 & 6.8 \\
\hline U14 & 40.7 & 12.8 & 42.7 & 62.6 & 5.9 \\
\hline U15 & 44.0 & 11.4 & 47.1 & 66.4 & 18.0 \\
\hline U16 & 41.8 & 12.3 & 43.0 & 64.4 & 12.3 \\
\hline U18 & 40.4 & 9.5 & 40.6 & 64.3 & 16.2 \\
\hline Overall & 40.2 & 11.2 & 41.1 & 66.4 & 5.9 \\
\hline
\end{tabular}

$\mathrm{U}=$ Under; $\mathrm{SD}=$ Standard Deviation. 
Table 2. Pairwise comparisons of the trial-to-trial reliability for the anterior Y-balance and back squat tests.

\begin{tabular}{|c|c|c|c|c|c|c|}
\hline & Trial 2 vs. Trial 1 & $90 \% \mathrm{CI}$ & Trial 3 vs. Trial 2 & $90 \% \mathrm{CI}$ & Overall & $90 \% \mathrm{CI}$ \\
\hline \multicolumn{7}{|c|}{ Anterior Y-balance Left } \\
\hline Mean change $(\%)$ & -1.8 & -4.4 to 0.85 & -3.2 & -6.3 to 0.01 & 2.7 & 2.4 to 3.0 \\
\hline Mean change $(\mathrm{cm})$ & -0.79 & -2.4 to 0.80 & -2.0 & -3.9 to -0.19 & 1.5 & 1.4 to 1.7 \\
\hline Qualitative Inference & Trivial & & Small & & Trivial & \\
\hline Typical error $(\%)$ & 8.8 & 7.7 to 10.4 & 10.8 & 9.4 to 12.7 & 9.8 & 8.8 to 11.2 \\
\hline Typical error (cm) & 5.1 & 4.5 to 6.0 & 5.8 & 5.1 to 6.8 & 5.5 & 4.9 to 6.2 \\
\hline Qualitative Inference & Moderate & & Large & & Large & \\
\hline ICC & 0.76 & 0.67 to 0.84 & 0.71 & 0.60 to 0.80 & 0.73 & 0.64 to 0.80 \\
\hline \multicolumn{7}{|c|}{ Anterior Y-balance Right } \\
\hline Mean change $(\%)$ & -1.3 & -4.5 to 2.1 & 2.9 & -0.14 to 6.1 & 2.3 & 2.0 to 2.6 \\
\hline Mean change $(\mathrm{cm})$ & -0.70 & -2.4 to 1.0 & 1.5 & -0.10 to 3.1 & 1.2 & 1.0 to 1.3 \\
\hline Qualitative Inference & Trivial & & Trivial & & Trivial & \\
\hline Typical error (\%) & 8.3 & 7.2 to 9.8 & 8.7 & 7.6 to 10.3 & 8.5 & 7.6 to 9.7 \\
\hline Typical error (cm) & 4.8 & 4.2 to 5.6 & 5.0 & 4.4 to 5.8 & 4.9 & 4.4 to 5.5 \\
\hline Qualitative Inference & Moderate & & Moderate & & Moderate & \\
\hline ICC & 0.79 & 0.70 to 0.86 & 0.77 & 0.68 to 0.84 & 0.78 & 0.70 to 0.84 \\
\hline \multicolumn{7}{|l|}{ Back squat } \\
\hline Mean change (\%) & 0.82 & -3.2 to 5.1 & 2.7 & -0.97 to 6.4 & 2.0 & 1.8 to 2.1 \\
\hline Mean change $(\mathrm{cm})$ & -0.19 & -1.4 to 1.1 & 0.70 & -0.37 to 1.8 & 0.51 & 0.48 to 0.56 \\
\hline Qualitative Inference & Trivial & & Trivial & & Trivial & \\
\hline Typical error $(\%)$ & 21.8 & 19.6 to 24.6 & 19.3 & 17.4 to 21.8 & 20.6 & 18.9 to 22.7 \\
\hline Typical error (cm) & 5.8 & 5.3 to 6.5 & 5.2 & 4.7 to 5.8 & 5.5 & 5.1 to 6.1 \\
\hline Qualitative Inference & Large & & Large & & Large & \\
\hline $\mathrm{ICC}$ & 0.75 & 0.68 to 0.80 & 0.77 & 0.71 to 0.83 & 0.76 & 0.70 to 0.81 \\
\hline Qualitative Inference & High & & High & & High & \\
\hline
\end{tabular}

ICC $=$ Intraclass correlation coefficient $\mathrm{CI}=$ confidence interval. 


\section{FIGURES}

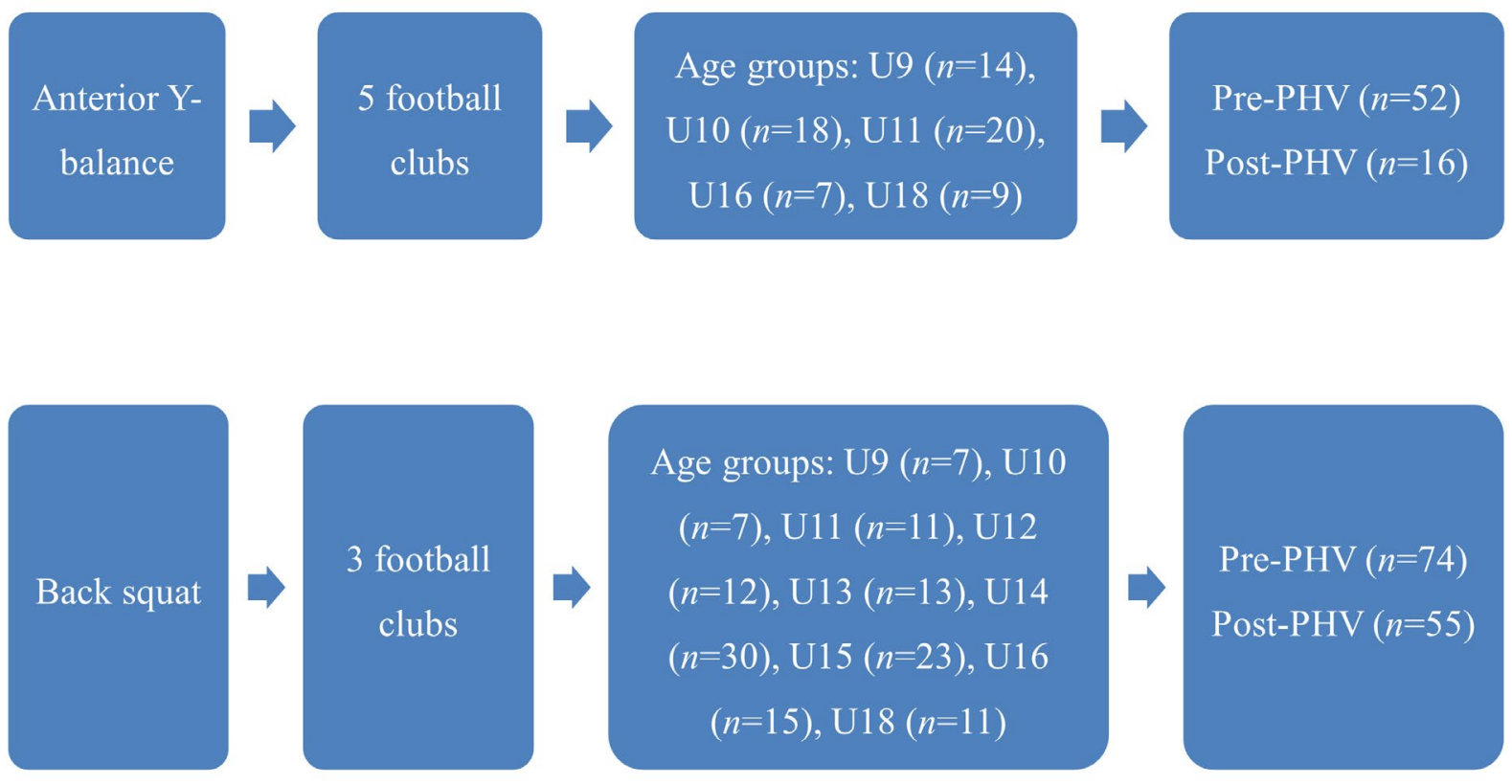

Figure 1. Scheme of allocation of the undertaken tests by chronological age and peak height velocity (PHV) across the football clubs. 

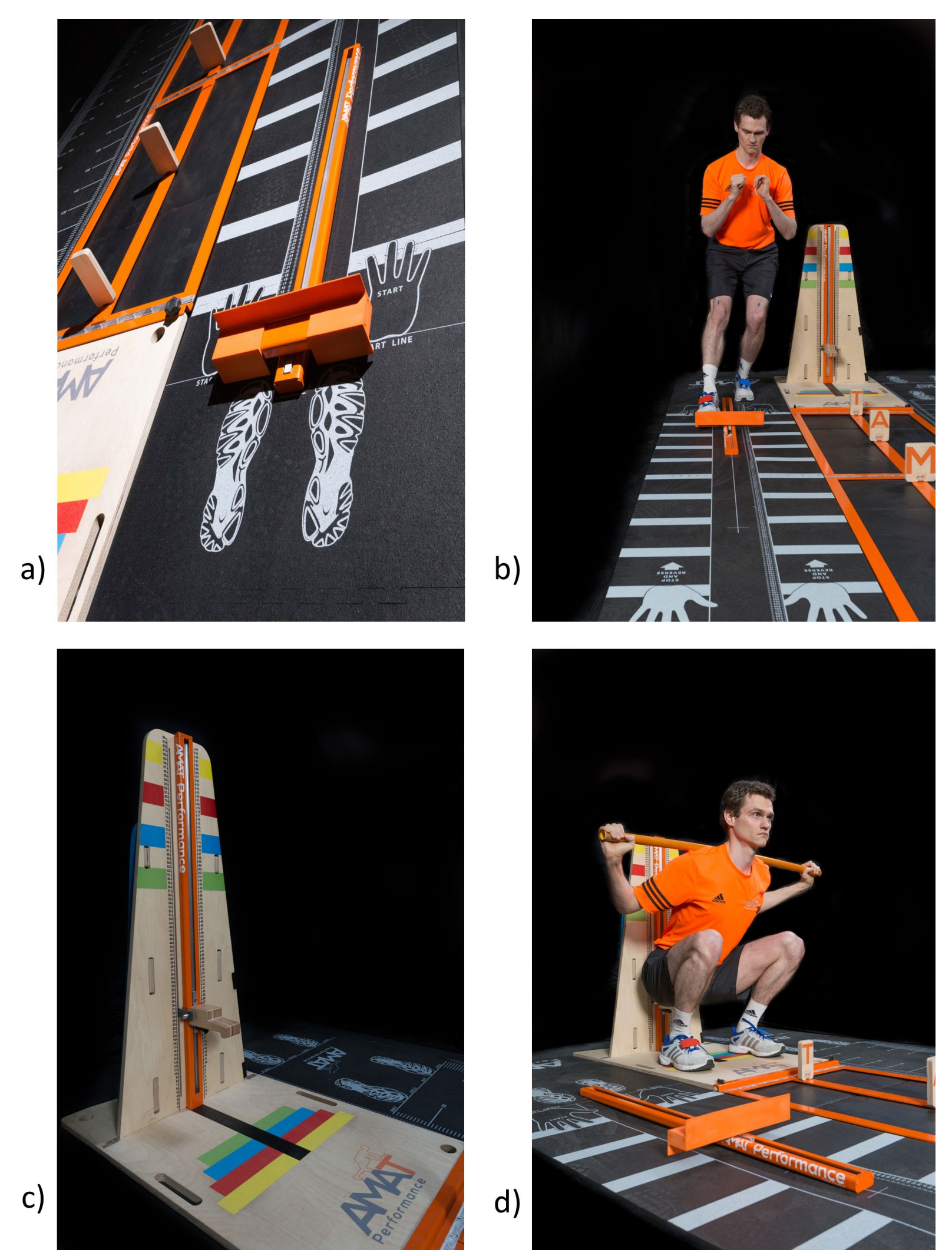

Figure 2. The slider (a) was used to measure the maximum reach distance for anterior Ybalance test (b) and squat saddle (c) was used to measure the maximum depth displacement in the back squat test (d). 

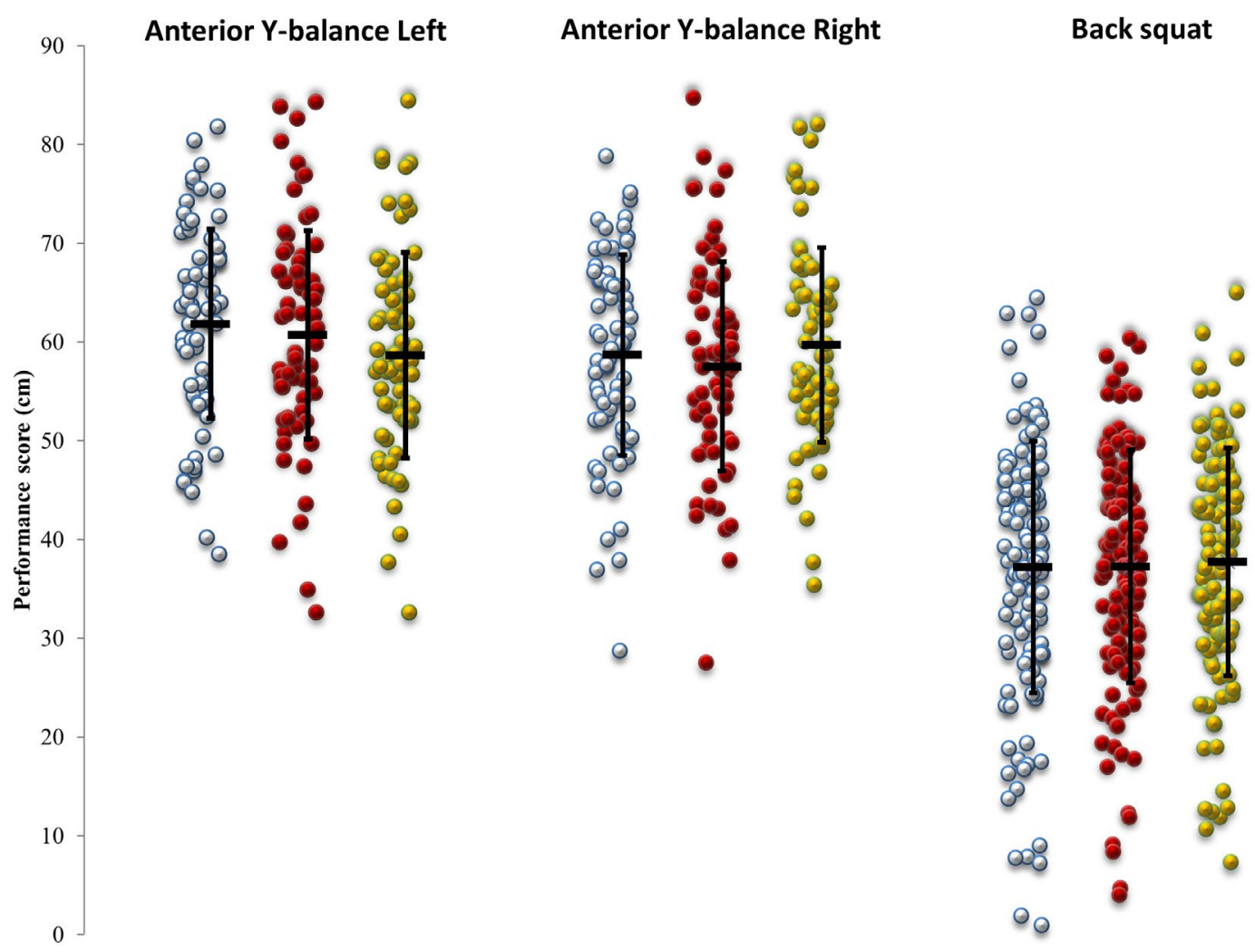

OTrial 1 Trial 2 Trial 3

Figure 3. Individual raw movement performance score $(\mathrm{cm})$ for all 3 trials for the anterior $\mathrm{Y}$ balance left, anterior Y-balance right and back squat tests. The horizontal solid line in the middle of the trials represents the mean and the vertical error bars standard deviations. 

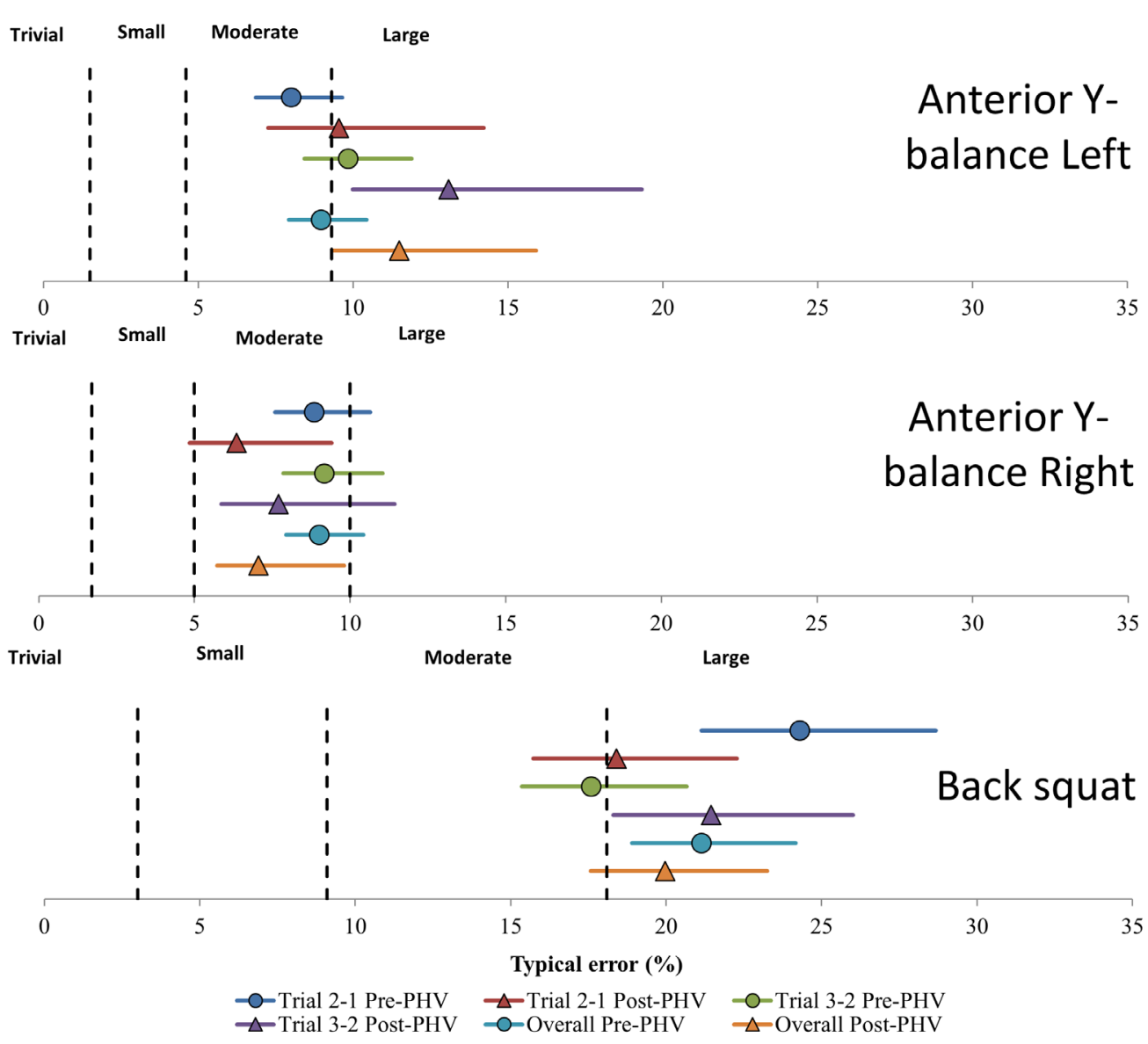

Figure 4. Between-trial typical errors (\%) for the anterior Y-balance left, anterior Y-balance right and back squat tests across peak height velocity (PHV). Solid horizontal lines represent 90\% confidence intervals. Dashed vertical lines represent thresholds for trivial, small, moderate and large effect sizes for the typical error. *A change in typical error across a threshold was considered meaningful (Hurst et al., 2018). 
